\title{
A Bioinspired Stretchable Sensory-Neuromorphic System
}

\section{Sun Hong Kim}

Seoul National University

\section{Geun Woo Baek}

Seoul National University

Jiyong Yoon

Sungkyunkwan University

\section{Seunghwan Seo}

Sungkyunkwan University

Donghyo Hahm

Sungkyunkwan University

Jun Hyuk Chang

Sungkyunkwan University

\section{Duhwan Seong}

Sungkyunkwan University

Hyunseon Seo

Korea Institute of Science and Technology

\section{Seyong Oh}

Sungkyunkwan University

\section{Kyunghwan Kim}

Seoul National University https://orcid.org/0000-0003-2243-6633

\section{Heeyoung Jung}

Seoul National University https://orcid.org/0000-0001-6094-3498

\section{Youngsu Oh}

Korea University https://orcid.org/0000-0002-6066-3151

\section{Hyoung Won Baac}

Sungkyunkwan University

\section{Batyrbek Alimkhanuly}

Kyunghee University

\section{Wan Ki Bae}

Sungkyunkwan University

\section{Seunghyun Lee}

Kyung Hee University Jeonghun Kwak 
Seoul National University https://orcid.org/0000-0002-4037-8687

Jin-Hong Park

Sungkyunkwan University https://orcid.org/0000-0001-8401-6920

Donghee Son ( $\nabla$ daniel3600@g.skku.edu )

Sungkyunkwan University https://orcid.org/0000-0002-3772-8009

\section{Article}

Keywords: neuromorphic, G-SSNS, QLED

Posted Date: September 21st, 2020

DOl: https://doi.org/10.21203/rs.3.rs-54383/v1

License: (c) (1) This work is licensed under a Creative Commons Attribution 4.0 International License. Read Full License

Version of Record: A version of this preprint was published at Advanced Materials on September 12th, 2021. See the published version at https://doi.org/10.1002/adma.202104690. 


\section{Abstract}

Conventional stretchable electronics entailing the adoption of a wavy design, a neutral mechanical plane, and a conformal contact between abiotic and biotic interfaces have shown diverse skin-interfaced applications. Despite such remarkable progress, there have been challenged to be evolved to intelligent skin prosthetics due to the absence of the monolithic integration of neuromorphic constituents into individual sensing and actuating components. Herein, we demonstrate a golden tortoise beetle-inspired stretchable sensory-neuromorphic system comprising an artificial mechanoreceptor, an artificial synapse, and an epidermal photonic actuator as three biomimetic functionalities that correspond to a stretchable capacitive pressure sensor, a resistive random-access memory, and a quantum dot light-emitting diode, respectively. This system features a rigid-island structure interconnected with a sinter-free printable conductor (stretchability 160\%, conductivity 18,550 S/cm), which allows one to improve both areal density and structural reliability while avoiding the thermal degradation of heat-sensitive stretchable electronic components. Moreover, even in the skin deformation range, the system accurately recognizes various patterned stimuli via an artificial neural network with training/inferencing functions. Our new bioinspired system is therefore expected to be an important step toward the implementation of intelligent wearable electronics.

\section{Introduction}

Stretchable electronic devices have been widely considered as individual modules for smart skin prosthetics owing to their exceptional electrical and mechanical reliability in dynamic environments; these features enable the monitoring of various external stimuli such as touch, heat, and humidity, and delivery of processed information to sensory nerves ${ }^{1-4}$. Although much effort has been directed the development of multifunctional systems comprising various active electronic components ${ }^{5-7}$, further progress in this field remains limited by the absence of the monolithic integration of neuromorphic constituents into individual sensing and actuating components. Thus, new methodology allowing one to overcome this insuperable problem is highly sought after.

As a novel alternative, one can consider a stretchable sensory-neuromorphic system. Again, skin-like electronic systems featuring self-adaptable data processability for unpredictable environments should exhibit various exceptional properties, such as i) mechanical matching in the integrated system ${ }^{8,9}$, ii) conformal contact for chronic high-quality signal acquisition ${ }^{10,11}$, iii) efficient pattern classification for improving sensing accuracy ${ }^{12}$, and iv) feedback-informative actuation in response to artificial synaptic potentials ${ }^{13,14}$. The application of artificial synapse modules to stretchable electronics, together with the monolithic integration, is inevitable for the realization of such unique sensory-neuromorphic systems. However, no such intelligent skin-like electronic systems have been reported to date.

In addition to the aforementioned unmet need, previously reported fabrication processes are of limited applicability to stretchable sensory-neuromorphic systems with high areal density, efficient integration, and high processing compatibility, largely because i) the rigid-island design based on wavy/buckled 
structures requires a substantial cell-to-cell distance to dissipate the accumulated strain energy through serpentine interconnections when stretched ${ }^{15-17}$ and ii) the low environmental stability, low throughput, low reproducibility, and complicated synthesis of intrinsically stretchable organic materials ${ }^{18-21}$ critically limit the maintenance of long-term reliable mechanical and electrical performance at biotic/abiotic interfaces. These issues primarily originate from the absence of both intrinsic stretchability of functional organic materials and the high-performance operation of inorganic air-stable thin film materials. Therefore, an optimal balance between the aforementioned approaches can result in a great breakthrough in the development of stretchable sensory-neuromorphic systems.

Herein, as a novel integrated platform, we fabricated a golden tortoise beetle-inspired stretchable sensoryneuromorphic system (G-SSNS) comprising an artificial mechanoreceptor, an artificial synapse, and an epidermal photonic actuator [constructed using a capacitive pressure sensor, a resistive random-access memory (RRAM), and a quantum dot light-emitting diode (QLED), respectively] as three vital parts. This system featured the bioinspired sequential operations of real-time tactual stimulus measurement, pattern learning/inferencing, and the visualization of feedback color transitions. The electrically reliable operation of the G-SSNS in the skin deformation range ( $25 \%$ strain) was realized by employing an effectively strain-dissipative design exploiting complementarily stretchable interconnects and rigid islands. Furthermore, the electrical stability of the G-SSNS was achieved by using an intrinsically stretchable conductive material (sinter-free printable conductor with a high stretchability of $\sim 160 \%$ and a high conductivity of $18,550 \mathrm{~S} / \mathrm{cm}$ ) as a strain-insensitive interconnect.

\section{Overall description of the G-SSNS}

The golden tortoise beetle (charidotella sexpunctata) has a unique function capable of sensing a gentle touch and changing color from gold to red (Fig. 1a) ${ }^{22-24}$. After recognizing external stimuli through mechanoreceptors and sensory nerves, the beetle's cognitive function controls the amount of fluid in the cuticle using the microscopic valve and thereby changing light reflection. The G-SSNS was able to emulate the sensory nervous system of the golden tortoise beetle using three vital functions, comprising i) an artificial mechanoreceptor for converting physical input into electrical potential, ii) an artificial synapse using a neural network based on training/inferencing, and iii) an epidermal photonic actuator for color change. These functionalities corresponded to a stretchable capacitive pressure sensor, a RRAM, and a QLED, respectively (Fig. 1b), with the sequential biomimetic functions being as follows. The artificial mechanoreceptor measured external stimuli in real time and transmitted the corresponding compressive stress information to the artificial synapse-powered neural network. The transmitted information was used by the neural network to perform training/inferencing via a back-propagation algorithm, and the inferenced information was delivered to the epidermal photonic actuator. Finally, the epidermal photonic actuator visualized the trained/inferenced information. Figure 1c (bottom left inset indicates the moment of gentle touch; top right inset is an image of a $5 \times 5$ QLED array) shows a representative image of the G-SSNS realized using conventional microfabrication, transfer-printing assembly, and stencil-printing processes. In particular, the replacement of a previously employed wavy/buckled interconnect with intrinsically stretchable printed wiring allowed for an efficient areal 
density and high deformability (Fig. 1c and Supplementary Fig. 1) ${ }^{17}$. More importantly, compared to conventional ink-printing methods accompanying thermal processes, our room-temperature ink-printing technique enabled the intrinsic electrical performances of the individual biomimetic modules to be made insusceptible to the thermal environment (Fig. 1c and Supplementary Fig. 2). The detailed properties of each bioinspired module are addressed below.

\section{Characterization of the intrinsically stretchable printed electrode and its application to the artificial mechanoreceptor}

The development of high-performance intrinsically stretchable interconnects is an essential prerequisite for mimicking the signal pathways in biological systems. Recent reports have inspired us to realize a sinter-free printable interconnect capable of forming a conductive percolation pathway under heating-free conditions owing to electron tunneling among in situ formed Ag nanoparticles around Ag flakes ${ }^{25-27}$. Thus, we developed an intrinsically stretchable printable conductor consisting of a dynamically crosslinked polymer [poly(dimethylsiloxane), 4,4'-methylenebis(phenyl urea), and isophorone bisurea (PDMS$\left.\left.\mathrm{MPU}_{0.4}-\mathrm{IU}_{0.6}\right)\right]$ and $\mathrm{Ag}_{\text {flakes }}{ }^{28}$. Generally, viscosity of the solution affects pattern resolution and scalability ${ }^{29}$. In this regard, three types of solvents [hexyl acetate $(\mathrm{HA})\left(\sim 160^{\circ} \mathrm{C}\right)$, methyl isobutyl ketone (MIBK) $\left(\sim 118^{\circ} \mathrm{C}\right)$, and chloroform $\left.\left(\sim 61^{\circ} \mathrm{C}\right)\right]$ were prepared to confirm the printability. After mixing with $\mathrm{Ag}$ flakes, each of sinter-free inks with different solvents was screen-printed on poly(dimethylsiloxane) substrates. The pattern widths of HA- and MIBK-based inks were almost identical to that $(500 \mu \mathrm{m})$ of the stencil mask (Supplementary Movie 1), while chloroform-based ink showed poor resolution (Supplementary Fig. 3a). The rapid evaporation of chloroform that has low boiling point causes pattern spreading when applying shear force to the ink. The HA-and MIBK-based ink show maximum printing resolution of $150 \mu \mathrm{m}$ (Supplementary Fig $3 \mathrm{~b}$ and 4).

Besides, Figure 2a shows the resistance change over 10 min after screen-printing (without sintering), revealing that the initial resistance of conductor positively correlated with the solvent boiling point. Hence, solvent-driven morphology was assumed to affect the formation of electrical percolative pathways in the printed composites. Similarly, the type of organic solvent also influenced composite mechanical properties. As shown in Fig. 2b, the MIBK-based conductor showed the highest conductivity of 18,550 $\mathrm{S} / \mathrm{cm}$ (at $50 \%$ strain) and a stretchability of $\sim 160 \%$ (Supplementary Movie 2), while the chloroformbased conductor showed the highest stretchability of $\sim 191 \%$ with a conductivity of $2,720 \mathrm{~S} / \mathrm{cm}$. Other inks showed similar stretchabilities of $\sim 160 \%$ and conductivities of $1,870 \mathrm{~S} / \mathrm{cm}(\mathrm{HA})$ and $9,990 \mathrm{~S} / \mathrm{cm}$ (MIBK) (Supplementary Fig. 5 and Supplementary Note 1, 2). The morphology of printed conductor was probed by the observation of Ag flake distribution by field-emission scanning electron microscopy (FESEM) (Fig. 2c, Supplementary Fig. 6 and Supplementary Note 2; insets of Fig. 2c show cross-sectional SEM images of each printed conductor). As the solvent boiling point decreased, Ag flakes became dispersive in the polymer matrix (Fig. 2c). Along this line, MIBK can be chosen as an optimal solvent because a formation of MIBK-driven optimal-sized clusters (aggregated Ag flakes) is effective in maintaining high conductivity while not causing severe crack propagations (Fig. 2d). Such electrical 
improvement is attributed to the fact that the $\mathrm{Ag}-\mathrm{Ag}$ interaction is more favorable than the polymer chain-Ag flake one ${ }^{30}$.

Interestingly, the conductivity of all three conductors increased with increasing strain in a small deformation range of up to $50 \%$ strain (conductivity at $50 \%$ strain: $\mathrm{HA}=7,720 \mathrm{~S} / \mathrm{cm}, \mathrm{MIBK}=18,550$ $\mathrm{S} / \mathrm{cm}$, chloroform $=5,870 \mathrm{~S} / \mathrm{cm}$ ). Moreover, the phenomenon, termed "electrical self-boosting", originates from the rearrangement of Ag flakes owing to the free volume of the strained polymer (Fig. 2e) ${ }^{25}$. The MIBK-based conductor showed the highest conductivity of $\sim 36,000 \mathrm{~S} / \mathrm{cm}$. This electrical self-boosting effect would be beneficial for realization of the strain-durable interconnect. An application of encapsulation layer to the soft conductor is also able to contribute to stretching durability. We encapsulated the MIBK-based conductor with PDMS (Fig. 2f), showing that no abrupt resistance change was observed until 1,000 cycles at $30 \%$ strain (Fig. $2 \mathrm{f}$ and Supplementary Fig. 5 and Supplementary Note 1). Optical microscopy images showed that after cyclic stretching, the morphology of the encapsulated surface was more uniform than that of the non-encapsulated one (Fig. $2 \mathrm{~g}$ ). Such encapsulation effect was well matched to the results in previous reports ${ }^{25,27,31}$. Based on the intrinsically stretchable printed conductor, the artificial mechanoreceptor ( $5 \times 5$ capacitive touch sensing array) was fabricated using sequential printing assemblies (Fig. 2h), which allowed one to significantly improve the cell density and scalability of the biomimetic sensor (Supplementary Fig. 7). Owing to the linearity of its relative capacitance change with the applied strain, the artificial mechanoreceptor recognized strain-induced deformation when stretched or pressed (Figs. 2i, j and Supplementary Note 3), while also showing high sensing reliability and uniformity (Fig. 2k and Supplementary Fig. 8).

\section{Characterization of the intrinsically stretchable RRAM and its application to the artificial synapse}

Despite the progress in the electronic skin systems for wearable and stretchable applications, the realization of smart skin systems is hindered by the absence of high-performance neuromorphic hardware/software modules involving precise and efficient recognition. To impart the synaptic functionality into skin-like systems, we used a RRAM module with an $\mathrm{Al} / \mathrm{TiO}_{2} / \mathrm{Al}$ layered structure as a neuromorphic device featuring cognitive computation in silico because of its low-power efficiency, high scalability, and stackability ${ }^{32-34}$. The RRAM cells fabricated on the $\mathrm{SiO}_{2} / \mathrm{Si}$ wafer were transfer-printed onto the stretchable substrate, and each island was then bridged by intrinsically stretchable printed interconnects (Fig. 3a, Supplementary Fig. 9, and Methods). Notably, our fabrication strategy enabled reliable deformability due to the efficient strain dissipation of the PDMS-MPU $\mathrm{P}_{0.4}-\mathrm{IU}_{0.6}$ polymer used in the interconnect while improving areal density 25,28 . However, the employed procedure featured some caveats associated with the printability of conductive ink on different surfaces and the contact resistance between the conductive ink and the evaporated metal film. Generally, the printing of MIBK-based inks onto polyimide (PI) substrates is hindered by the good wettability of the latter with the former. As ink spreading in our system had to be controlled to prevent short-circuiting, we modified the PI islands to reduce the ink-exposed area (Supplementary Fig. 9). After the application of the protruded pattern to the PI substrate, no spreading-related issues were observed during printing (Fig. $3 b$ ). The other issue, namely 
the contact resistance between Al and the conductive ink, was also carefully considered to prevent undesired power consumption. To make the interface between the artificial synapse and the interconnect more intimate, we applied an extended Au pad to the bottom Al layer (Fig. 3b and Supplementary Fig. 10), demonstrating that the contact resistance of the Al-Au interface was lower than that of the Al one. Using our fabrication strategy, we confirmed the bipolar resistive switching characteristics of the RRAM during stretching to up to $100 \%$ strain (Figs. 3c, d and Supplementary Note 3). Furthermore, the cyclic stretching endurance and data retention (inset of Fig. 3e) performances as well as cumulative uniformity were also stable for stretching to $25 \%$ strain, a value comparable to the skin deformation range (Fig. 3e and Supplementary Fig. 11). In addition, we validated the waterproof nature of the RRAM to prevent sweatinduced malfunction, revealing that the encapsulated RRAM immersed into phosphate-buffered saline (PBS) showed stable operation without significant current variation (Supplementary Fig. 12).

The memristive switching could also be applied to the artificial synapse, as the voltage-driven reversible migration of nonstoichiometric defects (oxygen vacancies or metal ions) in transition metal oxide switching materials is highly similar to that of transmitting ions (potassium or sodium) in synapses ${ }^{34}$. Basically, the dynamic behavior of oxygen vacancies/anions under an applied electric field plays a critical role in memristive switching governed by space-charge-limited and ohmic conduction ${ }^{35}$. Such transmitting agents can act as artificial neurotransmitters activating a postsynaptic neuron to trigger/inhibit action potentials. To confirm synaptic functionality, we evaluated the excitatory/inhibitory postsynaptic currents (EPSC/IPSC) and the long-term potentiation/depression (LTP/LTD) of the fabricated RRAM devices (Figs. 3f, g, h and Supplementary Fig. 13, 14). First, when a single excitatory $\left(V_{\text {top }}=-2.3 \mathrm{~V}, t_{\mathrm{d}}=20 \mathrm{~ms}\right.$, and $\left.V_{\text {read }}=-0.5 \mathrm{~V}\right)$ pulse was applied to the RRAM, the abruptly increased current flowing through the device gradually decreased to the initial level. This behavior originated from high electrical conduction based on trap filling that is biomimetically similar to EPSC involving the transient depolarization of the postsynaptic membrane potential (Fig. 3f). Furthermore, the opposite trend was observed when an inhibitory $\left(V_{\text {top }}=2.2 \mathrm{~V}, t_{\mathrm{d}}=100 \mathrm{~ms}\right.$, and $\left.V_{\text {read }}=0.5 \mathrm{~V}\right)$ pulse was applied to the RRAM. This reversible migration was possibly due to detrapped charges that are almost identical to the IPSC governing the repolarization induced by the flow of potassium cations out of the cell membrane (Fig. 3g). The related detailed data are presented in Supplementary Fig. 13. The aforementioned characteristics were sustained in the skin deformation range (stretching to up to $25 \%$ ) (Figs. $3 f$ and g). Additionally, we analyzed the LTP/LTD characteristics of the RRAM-based artificial synapse while applying a series of 100 excitatory/inhibitory pulses $\left(V_{\text {top }}=-2.3 \mathrm{~V} /+2.2 \mathrm{~V}\right.$ and $\left.t_{\mathrm{d}}=20 \mathrm{~ms} / 100 \mathrm{~ms}\right)$ (Fig. $3 \mathrm{~h}$ and Supplementary Fig. 14), observing typical LTP/LTD properties after persistent strengthening and weakening due to the application of artificial action potentials. Such synaptic performances were also maintained in the $25 \%$-stretched state (Fig. 3h and Supplementary Table 1).

\section{Characterization of intrinsically stretchable QLEDs and their application to the epidermal photonic actuator}


The golden tortoise beetle employs a unique defense mechanism ${ }^{36,37}$ entailing a structural change of its cuticle. Specifically, the beetle can modulate the refractive index and thickness of its cuticle by controlling the amount of water in its epidermis. The transformed structure of the epidermis allows the beetle to modify the wavelength of the reflected light, which can be almost identical to that reflected by nearby surfaces. Similarly, we adopted colloidal quantum dots (QDs) as the core material of the epidermal photonic actuator owing to their core size-dependent tunable color ${ }^{38-40}$, demonstrating that the QD-based epidermis visualizes the trained/inferenced information transmitted by artificial synapses. A realization of the QD-based epidermal photonic actuator began with the fabrication of an inverted top emission QLED as the epidermal photonic actuator of the G-SSNS (Fig. 4a). The inverted top emission structure was not only required to display information to observers but also allowed one to minimize light extraction losses caused by the waveguide effect. The fabricated device featured a PDMS/PI/Ag/ZnO $\mathrm{NPs} / \mathrm{QDs} / 4,4$ '-bis(9-carbazolyl)-biphenyl) (CBP)/molybdenum oxide $\left(\mathrm{MoO}_{\chi}\right) / \mathrm{Ag} / \mathrm{SiO}_{2}$ structure. $\mathrm{Au}$ auxiliary electrodes were deposited to reduce the contact resistance between the QLED electrodes and the sinter-free stretchable interconnects, as shown in Fig. 4a and Supplementary Fig. 15, with the complete procedures described in Supplementary Fig. 16 and Supplementary Note 4. As aforementioned, the sinterfree interconnect could minimize the change of oxygen vacancies in $\mathrm{ZnO}$ and the molecular degradation in CBP possessing poor thermal properties (i.e., glass transition temperature $\approx 62^{\circ} \mathrm{C}$ ), enabling highly uniform and stable epidermal photonic actuation in the G-SSNS ${ }^{41-43}$.

In addition to offering structural benefits, colloidal QDs were optimized via ligand modification to control the electron-hole balance difference and minimize the injection barrier between the QDs and the adjacent layer. In particular, the long oleic acid (OA) molecules were replaced with shorter octylamine (OcA) ones to shorten the QD-to-QD distance and thus minimize the insulation property and control the energy level shift without changing the bandgap 44,45 . The effect of ligand exchange was validated using Fourier transform infrared spectroscopy (FTIR), which showed that after ligand exchange, the $\mathrm{N}-\mathrm{H}$ stretch was observed at $3200-3500 \mathrm{~cm}^{-1}$. In addition, photoluminescence quantum yield (PLQY) of the OcA-bearing QD solution (88\%) exceeded that of the OA-bearing QD solution (80\%) (Supplementary Fig. 17). To compare the performances of devices with OA- and OcA-capped QLEDs, we recorded the current densityluminance-voltage $(J-V-L)$ characteristics, external quantum efficiency (E.Q.E), and normalized electroluminescence (EL) spectra on a rigid glass substrate (Figs. 4b, c, d, Supplementary Fig. 18, Supplementary Note 5 and Methods). The current density of OcA-capped QLEDs was higher than that of OA-capped QLEDs, and the maximum luminance and E.Q.E of the former were 1.8- and 1.5-fold higher than those of the latter, respectively. The full width at half maximum of OcA- and OA-capped devices was determined from their normalized EL spectra as $38 \mathrm{~nm}$ (Supplementary Table 2). These properties, enhanced by the lowered hole barrier between QDs and CBP, were further confirmed by ultraviolet photoelectron spectroscopy (Fig. 4e and Supplementary Fig. 19). Furthermore, to ascertain whether the modification of QD ligands affects device performance, we compared electron-only devices (EODs) with hole-only devices (HODs) (Supplementary Fig. 20). The hole current of HODs with OcA-capped QDs was seven-fold higher than that of HODs with OA-capped QDs, which resulted in an improved electron-hole balance in the corresponding QLEDs. In addition, our sinter-free printing technique presented a crucial 
advantage for QLEDs, allowing one to sustain device performance without thermal degradation that can be caused by sintering. In a control test, a decreased E.Q.E and an increased driving voltage were observed when QLEDs were exposed to thermal stress for $1 \mathrm{~h}$ at $70^{\circ} \mathrm{C}$ (Supplementary Fig. 21).

The optimized epidermal photonic actuator based on QLEDs showed reliable mechanical properties upon crumpling and stretching to up to $25 \%$ strain (Figs. $4 \mathrm{f}, \mathrm{g}$ and Supplementary Movie 3 ) and could also sustain reliability in cyclic test of $10 \%$ strain without any remarkable luminance change (Supplementary Fig. 22). Interestingly, the luminance values of OA- and OcA-capped QLEDs were slightly increased (OAcapped QLED, $215 \mathrm{~cd} / \mathrm{m}^{2}$ at $0 \%$ strain and $242 \mathrm{~cd} / \mathrm{m}^{2}$ at $25 \%$ strain; OcA-capped QLED, $322 \mathrm{~cd} / \mathrm{m}^{2}$ at 0 $\%$ strain and $342 \mathrm{~cd} / \mathrm{m}^{2}$ at $25 \%$ strain) when stretched up to $25 \%$ strain. Such photonic improvement is possibly due to the rearrangement of $\mathrm{Ag}$ flakes in the free volume of the strained PDMS-MPU $0.4-\mathrm{IU}_{0.6}$ polymer ${ }^{46,47}$. To probe the stability of our device to perspiration, we measured its $J-V-L$ characteristics upon exposure to PBS with a pH of 7.2 (Figs. $4 \mathrm{~h}$ and i), revealing that the $\mathrm{SiO}_{2} / \mathrm{PDMS}$ encapsulation layers could effectively block sweat absorption, as expected.

\section{Demonstration of G-SSNS operation}

We then demonstrated sequentially the operation of the G-SSNS including real-time sensing, learning/inferencing, and feedback visualization, as illustrated in Fig. 5a. The G-SSNS received patterned stimuli through the artificial mechanoreceptors based on capacitive pressure sensors and then transmitted the voltage signals to the hardware neural network consisting of RRAM synapses. After training the neural network with patterned stimuli, the signals obtained from this network through inference process were transmitted to the epidermal photonic actuator, which emitted patterned lights corresponding to localized stimuli (Supplementary Fig. 23). The G-SSNS was evaluated in terms of the aforementioned signal processing, especially training and inferencing, under two distinct conditions, namely in pristine and $25 \%$-stretched states (Fig. $5 \mathrm{~b}$ ). The results demonstrated the self-adaptable data processability of the G-SSNS for unpredictable environments under deformation-induced electrical disturbance. As aforementioned, the system received four kinds of patterned stimuli ("S," "N," "U," and "K") through a $5 \times 5$ capacitive pressure sensor array as an artificial mechanoreceptor. These stimuli were then transformed into the image patterns, each pixel of which presented a voltage signal $\left(V_{\mathrm{n}}\right)$. Figure $5 \mathrm{c}$ shows the image patterns obtained from pristine (top) and $25 \%$-stretched (bottom) sensor arrays, respectively (Supplementary Fig. 24), where the latter patterns presented larger voltage signals than the former ones (0.15-4.28 $\mathrm{V}$ for pristine and 0.18-4.97 $\mathrm{V}$ for $25 \%$-stretched arrays). The capacitance values measured by placing 3D printed objects on the artificial mechanoreceptor were converted to voltages using custom-made software (Supplementary Fig. 25 and Supplementary Note 6). The voltage signals corresponding to each pixel were applied to the designed neural network consisting of 25 input neurons, 4 output neurons, and $25 \times 4$ synapses connected to the neurons. The pristine and $25 \%$ stretched RRAM synapses were employed in two different neural networks depicted in Fig. $5 \mathrm{~d}$-the left one was unaffected by deformation-based disturbance, while the right one was affected. For training the neural networks, the synapse weights were updated using a back-propagation algorithm (details are 
presented in Supplementary Note 7 as well as Tables 3 and 4). For inferencing, the applied voltage signals were multiplied by synapse weights $\left(W_{n, m}\right)$ and summed at the output neurons, where the synapse weight was defined as synapse conductance $(W=G)$ (see also Supplementary Fig. 26). Consequently, the output currents ( ) obtained at the output neuron layer were transformed using a sigmoid activation function to afford output neuron signals $\left(f_{\mathrm{m}}\right)$. Figures $5 \mathrm{e}$ and $\mathrm{f}$ show the weight mapping and synaptic weight values connected to $f_{1, s}$ pattern with respect to the training epoch (Supplementary Fig. 27). With progressing the training, the weights of pristine and $25 \%$-stretched synapses were gradually potentiated or depressed, reaching values as high as 180 and $944 \mu \mathrm{S}$ or as low as $42 \mu \mathrm{S}$ and $1.72 \mathrm{mS}$, respectively. The pattern recognition efficiency and the number of misclassified patterns were then calculated for every 10 epochs, as shown in Fig. 5g. After 140 epochs, a $100 \%$ pattern recognition efficiency and no misclassified patterns were observed under both pristine and $25 \%$-stretched conditions, which demonstrated stable data processability under deformation-based disturbance. Finally, as depicted in Fig. 5h, we successfully visualized feedback actuation via the $5 \times 5$ QLED arrays in response to inference results (for " $\mathrm{S}$," "N," " $\mathrm{U}$," and "K" patterns), in which case the output voltage signals of the neural networks were transmitted to the epidermal photonic actuator (see also Supplementary Figs. 28, 29, Supplementary Note 8 and Supplementary Movie 4).

\section{Conclusions}

Inspired by the excellent touch-induced color-tunable ability of the golden tortoise beetle, we realized a biomimetic multifunctional stretchable sensory-neuromorphic system comprising an artificial mechanoreceptor, an artificial synapse, and an epidermal photonic actuator. These bioinspired sensoryneuromorphic functions were made accessible even in deformation modes by using a rational combination of intrinsically stretchable printed interconnects and high-performance rigid-island active cells as well as self-adaptable data processability. The trained/inferenced information was then visualized using the photonic functionality. The new fabrication and integration approaches presented herein are expected to facilitate the paradigm change of smart skin prosthetic systems.

\section{Methods}

Sinter-free ink and capacitance sensor fabrication. To formulate the sinter-free ink, the polymer (PDMS$\mathrm{MPU}_{0.4}-\mathrm{IU}_{0.6}$ ) and MIBK (Sigma Aldrich) were mixed for $1 \mathrm{~h}$, and the obtained solution was mixed with Ag flakes (DSF-500MWZ-S, Daejoo Electronics) and stirred for $5 \mathrm{~h}$ (Ag flake:polymer $=4: 1, \mathrm{w} / \mathrm{w}$ ). The bottom electrodes of the capacitance sensor were fabricated by screen-printing using a metal screen mask (width $=500 \mu \mathrm{m})$ and a waiting time of $30 \mathrm{~min}$ was required for stabilization of sinter-free conductor resistances. The dielectric layer was screen-printed on the bottom electrode using a square metal screen mask and a waiting time of $24 \mathrm{~h}$ was required. Finally, the top electrode was fabricated by screen-printing of sinter-free ink. 
Fabrication of the resistive RRAM array. The fabrication of the RRAM array is described in detail in Supplementary Fig. 9. The PI precursor solution (polyamic acid, Sigma Aldrich) was spin-coated at 3000 rpm for $60 \mathrm{~s}$ on the wafer, softly cured at $150{ }^{\circ} \mathrm{C}$ for $15 \mathrm{~min}$, and fully cured at $250{ }^{\circ} \mathrm{C}$ for $1 \mathrm{~h}$. $\mathrm{Cr} / \mathrm{Au}(7$ $\mathrm{nm} / 60 \mathrm{~nm}$ ) layers were deposited by thermal evaporation and patterned by photolithography and wet etching to afford a bottom contact electrode for interconnecting the ink. An Al $(60 \mathrm{~nm})$ bottom electrode was thermally deposited and patterned by photolithography. $\mathrm{A} \mathrm{TiO}_{2}$ layer ( 50 -nm-thick) was then deposited by radio frequency magnetron sputtering at a base pressure of $5 \times 10^{-6} \mathrm{Torr}$, room temperature, a deposition pressure of $5 \mathrm{mTorr}, 20 \mathrm{sccm}$, and an RF power of $150 \mathrm{~W}$. Al/Au $(60 \mathrm{~nm} / 60 \mathrm{~nm})$ was thermally deposited as a top contact electrode using a shadow mask. To fabricate the encapsulation layer, an epoxy resin (SU-8, Microchem, USA) was spin-coated at $3000 \mathrm{rpm}$ for $60 \mathrm{~s}$, soft-cured, and patterned using photolithography. The bottom $\mathrm{PI}$ layer was then subjected to reactive ion etching using $\mathrm{O}_{2}$ plasma $\left(\mathrm{O}_{2}\right.$ flow rate $=100 \mathrm{sccm}$, chamber pressure $=100 \mathrm{mTorr}$, RF power $=150 \mathrm{~W}$ for $\left.7 \mathrm{~min}\right)$ to define PI islands.

Fabrication of the top emissive QLED on glass substrate. An Ag $(85 \mathrm{~nm})$ bottom cathode layer was thermally deposited using a metal mask. ZnO NPs dissolved in 1-butanol $(20 \mathrm{mg} / \mathrm{mL})$ were spin-coated at $2000 \mathrm{rpm}$ for $40 \mathrm{~s}$ and baked at $100{ }^{\circ} \mathrm{C}$ for $30 \mathrm{~min}$ at $\mathrm{N}_{2}$ atmosphere. Then, a $25 \mathrm{mg} / \mathrm{mL}$ dispersion of QDs in chlorobenzene was spin-coated at $4000 \mathrm{rpm}$ for $30 \mathrm{~s}$ and annealed at $90{ }^{\circ} \mathrm{C}$ for 30 min under $\mathrm{N}_{2}$. Subsequently, $\mathrm{CBP}(60 \mathrm{~nm}) / \mathrm{MoO}_{\mathrm{x}}(10 \mathrm{~nm}) / \mathrm{Ag}(20 \mathrm{~nm})$ layers were deposited by thermal evaporation using a metal shadow mask. Details of fabrication process of intrinsically stretchable QLEDs are shown in Supplementary Fig. 16 and Supplementary Note 4.

Materials for QD synthesis. Zinc acetate (Zn(ac)2, $99.99 \%)$, sulfur (S, $99.998 \%$ ), selenium (Se, $99.99 \%)$, oleic acid (OA, $99 \%$ ), and 1-octadecene (ODE, $99 \%$ ) were purchased from UniAm (Korea). n-

Trioctylphosphine (TOP, technical grade, $90 \%$ ) was purchased from Sigma Aldrich. Cadmium oxide (CdO, $99.95 \%$ ), myristic acid (MA, >99\%) and 1-dodecanethiol (DDT, >98\%) were purchased from Alfa Aesar. All chemicals are used as received without further purification.

Precursors for QD synthesis. A 0.5 M cadmium oleate $\left(\mathrm{Cd}(\mathrm{OA})_{2}\right)$ stock solution, a $0.5 \mathrm{M}$ zinc oleate $\left(\mathrm{Zn}(\mathrm{OA})_{2}\right)$ stock solution, a $2 \mathrm{M}$ TOPSe solution, and a $2 \mathrm{M}$ TOPS solution were prepared as cation and anion precursors, respectively. To prepare the $\mathrm{Cd}(\mathrm{OA})_{2}$ stock solution, $\mathrm{CdO}(30 \mathrm{mmol}), \mathrm{OA}(30 \mathrm{~mL})$, and ODE $(30 \mathrm{~mL})$ were loaded in a reaction flask and degassed at $110^{\circ} \mathrm{C}$ for $1 \mathrm{~h}$. The flask was backfilled with $\mathrm{Ar}$, heated to $300^{\circ} \mathrm{C}$ to obtain a clear $\mathrm{Cd}(\mathrm{OA})_{2}$ solution, cooled to $110^{\circ} \mathrm{C}$, and degassed for $1 \mathrm{~h}$. To prepare the $\mathrm{Zn}(\mathrm{OA})_{2}$ stock solution, $\mathrm{Zn}(\mathrm{ac})_{2}(100 \mathrm{mmol})$ and $\mathrm{OA}(100 \mathrm{~mL})$ were placed in a flask and degassed for $1 \mathrm{~h}$. After degassing, the reaction temperature was raised to $160{ }^{\circ} \mathrm{C}$ and maintained until the formation of the $\mathrm{Zn}(\mathrm{OA})_{2}$ solution was complete. The flask was filled with $\mathrm{Ar}$, and the solution was diluted with degassed ODE to a concentration of $0.5 \mathrm{M}$. To prepare $2 \mathrm{M}$ TOPS and $2 \mathrm{M}$ TOPSe stock solutions, $\mathrm{S}$ or Se $(50 \mathrm{mmol})$, respectively, was dissolved in TOP $(25 \mathrm{~mL})$ at an elevated temperature. 
Synthesis of red-light-emitting CdSe/CdZnS QDs. All reactions were conducted in an inert atmosphere using Schlenk techniques. In a typical synthesis of CdSe/CdZnS QDs, a mixture of CdO (1 mmol), MA (3 $\mathrm{mmol})$, and $\mathrm{ODE}(15 \mathrm{~mL})$ was degassed in a flask at $110^{\circ} \mathrm{C}$ under vacuum. After degassing, the flask was backfilled with $\mathrm{Ar}$, and the temperature was raised to $270^{\circ} \mathrm{C}$. Then, TOPSe $(0.5 \mathrm{mmol})$ was rapidly injected into the flask to promote the nucleation of CdSe cores. After $3 \mathrm{~min}$, the flask was charged with the $\mathrm{Zn}(\mathrm{OA})_{2}$ solution $(4 \mathrm{~mL})$, and DDT $(1.5 \mathrm{mmol})$ was dropwise added to grow the CdZnS shell. After 30 min, $\mathrm{Cd}(\mathrm{OA})_{2}, \mathrm{Zn}(\mathrm{OA})_{2}$, and TOPS were repeatedly injected for the additional growth of the $\mathrm{CdZnS}$ shell to achieve a total shell thickness of $4.5 \mathrm{~nm}$. The synthesized CdSe/CdZnS QDs were repeatedly purified by a typical precipitation/ redispersion method.

\section{Declarations}

\section{Acknowledgements}

This work was supported by the National Research Foundation of Korea (NRF) grant funded by the Korea government (MSIT) (No. 2020R1C1C1005567/ No. 2018R1A2A2A05010475) and Institute of Information \& communications Technology Planning \& Evaluation (IITP) grant funded by the Korea government (MSIT) (No.S-2020-1443-000, Development of low power/low delay/self-power suppliable RF simultaneous information and power transfer system and stretchable electronic epineurium for wireless nerve bypass implementation). This work was also supported by the Industrial Core Technology Development Program (10077471) funded by the Ministry of Trade, Industry and Energy of Korea.

\section{Author contributions}

D.S. proposed the project. D.S, J.-H.P, and J.K. supervised the project. S.H.K., G.W.B., J.Y. conducted all experiments with assistance from S.S., D.H., J.H.C., D.S., H.S., S.O., K.K., J.H., Y.O., H.Y., B.A., W.K.B., S.Y., S.H.K., G.W.B., J.Y., S.S., J.K., J.-H.P., and D.S. analyzed the data and wrote the manuscript with input from all coauthors.

\section{Competing interests}

The authors declare no competing financial interests.

\section{References}

1. Kim, Y. et al.A bioinspired flexible organic artificial afferent nerve. Science360, 998-1003 (2018).

2. Shim, H. et al. Stretchable elastic synaptic transistors for neurologically integrated soft engineering systems. Adv. 5, eaax4961 (2019).

3. Zhao, H., O'Brien, K., Li, S. \& Shepherd, R. F. Optoelectronically innervated soft prosthetic hand via stretchable optical waveguides. Robot.1, eaai7529 (2016).

4. Kim, D.-H. et al. Epidermal electronics. Science333, 838-843 (2011). 
5. Park, H.-L. et al. Flexible neuromorphic electronics for computing, soft robotics, and neuroprosthetics. Mater. 32, 1903558 (2020).

6. Son, D. et al. Multifunctional wearable devices for diagnosis and therapy of movement disorders. Nanotech.9, 397-404 (2014).

7. Huang, Z. et al. Three-dimensional integrated stretchable electronics. Electron. 1, 473-480 (2018).

8. Dagdeviren, C. et al. Conformal piezoelectric systems for clinical and experimental characterization of soft tissue biomechanics. Mater.14, 728-736 (2015).

9. Yuk, H. et al. Dry double-sided tape for adhesion of wet tissues and devices. Nature575, 169-174 (2019).

10. Someya, T. \& Amagai, M. Toward a new generation of smart skins. Biotechnol.37, 382-388 (2019).

11. Sugiyama, M. et al. An ultraflexible organic differential amplifier for recording electrocardiograms. Electron.2, 351-360 (2019).

12. Seo, S. et al. Artificial optic-neural synapse for colored and color-mixed pattern recognition. Commun.9, 5106 (2018).

13. Lee, Y. et al. Stretchable organic optoelectronic sensorimotor synapse. Adv.4, eaat7387 (2018).

14. Chou, $\mathrm{H}$. et al. A chameleon-inspired stretchable electronic skin with interactive colour changing controlled by tactile sensing. Commun.6, 8011 (2015).

15. Hua, Q. et al. Skin-inspired highly stretchable and conformable matrix networks for multifunctional sensing. Commun.9, 244 (2018).

16. Xu, S. et al. Soft microfluidic assemblies of sensors, circuits, and radios for the skin. Science344, 7074 (2014).

17. Wang, C. et al. Soft ultrathin electronics innervated adaptive fully soft robots. Mater.30, 1706695 (2018).

18. Liang, J. et al. Intrinsically stretchable and transparent thin-film transistors based on printable silver nanowires, carbon nanotubes and an elastomeric dielectric. Nat Commun.6, 7647 (2015).

19. Mun, J. et al. Conjugated carbon cyclic nanorings as additives for intrinsically stretchable semiconducting polymers. Mater.31, 1903912 (2019).

20. Park, M. et al. Highly stretchable electric circuits from a composite material of silver nanoparticles and elastomeric fibres. Nanotech.7, 803-809 (2012).

21. Lei, Z. \& Wu, P. A highly transparent and ultra-stretchable conductor with stable conductivity during large deformation. Commun.10, 3429 (2019).

22. Sato, H. et al. Remote radio control of insect flight. Integr. Neurosci.3, 24 (2009).

23. Budick, S.A., Reiser, M.B. \& Dickinson, M.H. The role of visual and mechanosensory cues in structuring forward flight in Drosophila melanogaster. Exp. Biol. 210, 4092-4103 (2007).

24. Burrows, M. The Neurobiology of an Insect Brain (Oxford Univ. Press, 1996).

25. Kim, S.H. et al. An ultrastretchable and self-healable nanocomposite conductor enabled by autonomously percolative electrical pathways. ACS Nano 13, 6531-6539 (2019). 
26. Suh, D. et al. Electron tunneling of hierarchically structured silver nanosatellite particles for highly conductive healable nanocomposites. Commun.11, 2252 (2020).

27. Matsuhisa, N. et al. Printable elastic conductors by in situ formation of silver nanoparticles from silver flakes. Mater.16, 834-840 (2017).

28. Kang, J. et al. Tough and water-insensitive self-healing elastomer for robust electronic skin. Mater.30, 1706846 (2018).

29. Liang, J., Tong, K. \& Pei, Q. A water-based silver-nanowire screen-print ink for the fabrication of stretchable conductors and wearable thin-film transistors. Mater.28, 5986-5996 (2016).

30. Lopes, W. \& Jaeger, H. Hierarchical self-assembly of metal nanostructures on diblock copolymer scaffolds. Nature414, 735-738 (2001).

31. Matsuhisa, N. et al. Printable elastic conductors with a high conductivity for electronic textile applications. Commun.6, 7461 (2015).

32. van de Burgt, Y. et al. A non-volatile organic electrochemical device as a low-voltage artificial synapse for neuromorphic computing. Mater.16, 414-418 (2017).

33. Pickett, M., Medeiros-Ribeiro, G. \& Williams, R. A scalable neuristor built with Mott memristors. Mater.12, 114-117 (2013).

34. Xia, Q. \& Yang, J.J. Memristive crossbar arrays for brain-inspired computing. Mater.18, 309-323 (2019).

35. Kim, S. \& Choi, Y. A comprehensive study of the resistive switching mechanism in $\mathrm{Al} / \mathrm{TiO}_{\mathrm{x}} / \mathrm{TiO}_{2} / \mathrm{Al}-$ structured RRAM. IEEE Trans. Electron. Dev.56, 3049-3054 (2009).

36. Zhao, Y., Xie, Z., Gu, H., Zhu, C. \& Gu, Z. Bio-inspired variable structural color materials. Soc. Rev. 41, 3297-3317 (2012).

37. Vigneron, J.P. et al. Switchable reflector in the Panamanian tortoise beetle Charidotella egregia (Chrysomelidae: Cassidinae). Rev. E76, 031907 (2007).

38. Kwak, J. et al. Bright and efficient full-color colloidal quantum dot light-emitting diodes using an inverted device structure. Nano Lett.12, 2362-2366 (2012).

39. Li, X. et al. Bright colloidal quantum dot light-emitting diodes enabled by efficient chlorination. Photon.12, 159-164 (2018).

40. Kim, J. et al. Ultrathin quantum dot display integrated with wearable electronics. Mater.29, 1700217 (2017).

41. Kim, S.H. et al. Ultrastretchable conductor fabricated on skin-like hydrogel-elastomer hybrid substrates for skin electronics. Mater.30, 1800109 (2018).

42. Zeng, $\mathrm{H}$. et al. Blue luminescence of $\mathrm{ZnO}$ nanoparticles based on non-equilibrium processes: defect origins and emission controls. Funct. Mater.20, 561-572 (2010).

43. Tsai, M.-H. et al. 3-(9-Carbazolyl)carbazoles and 3,6-Di(9-carbazolyl)carbazoles as effective host materials for efficient blue organic electrophosphorescence. Mater.19, 862-866 (2007). 
44. Wang, R. et al. Colloidal quantum dot ligand engineering for high performance solar cells. Energy Environ. Sci.9, 1130-1143 (2016).

45. Shen, H. et al. High-efficiency, low turn-on voltage blue-violet quantum-dot-based light-emitting diodes. Nano Lett.15, 1211-1216 (2015).

46. Kim, T.-H. et al. Fully stretchable optoelectronic sensors based on colloidal quantum dots for sensing photoplethysmographic signals. ACS Nano11, 5992-6003 (2017).

47. Li, Y.-F. et al. Stretchable organometal-halide-perovskite quantum-dot light-emitting diodes. Mater.31, 1807516 (2019).

\section{Figures}


a

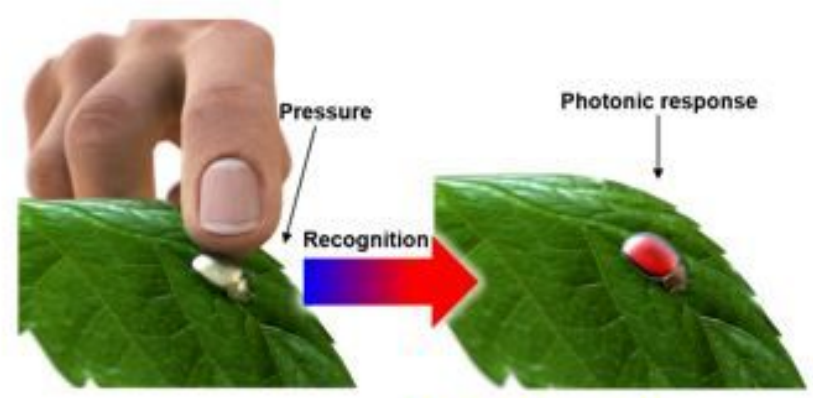

Mechanoreceptor Receptor potential

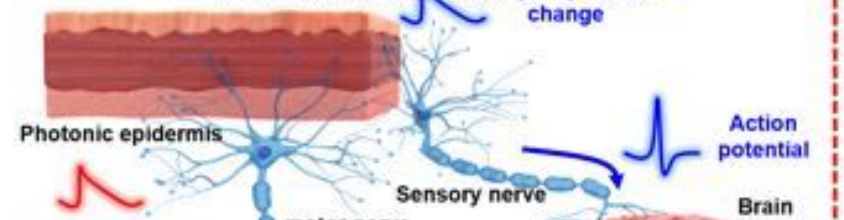

Receptor potential change b

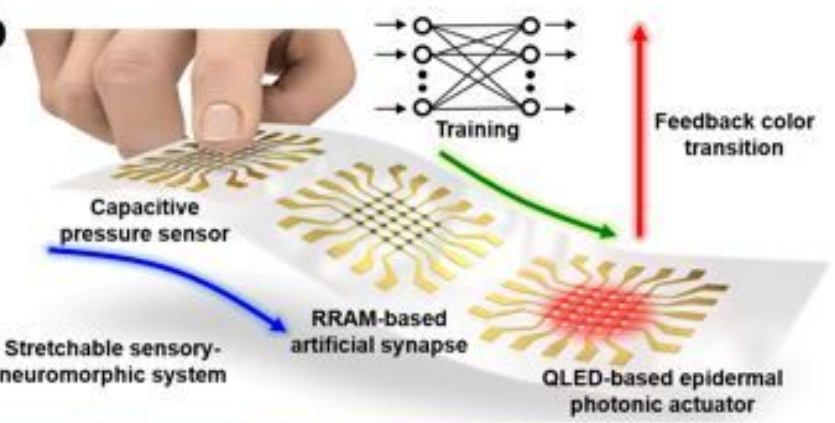
photonic actuator

QLED-based epidermat photonic actuator
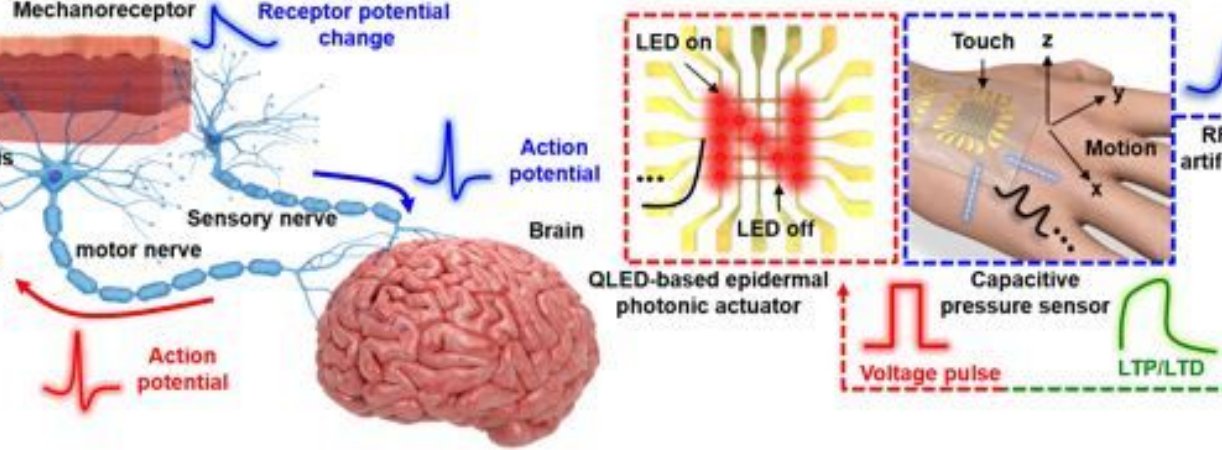

Voltage pulse s.

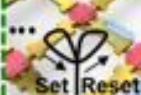

Capacitance change

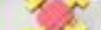

C

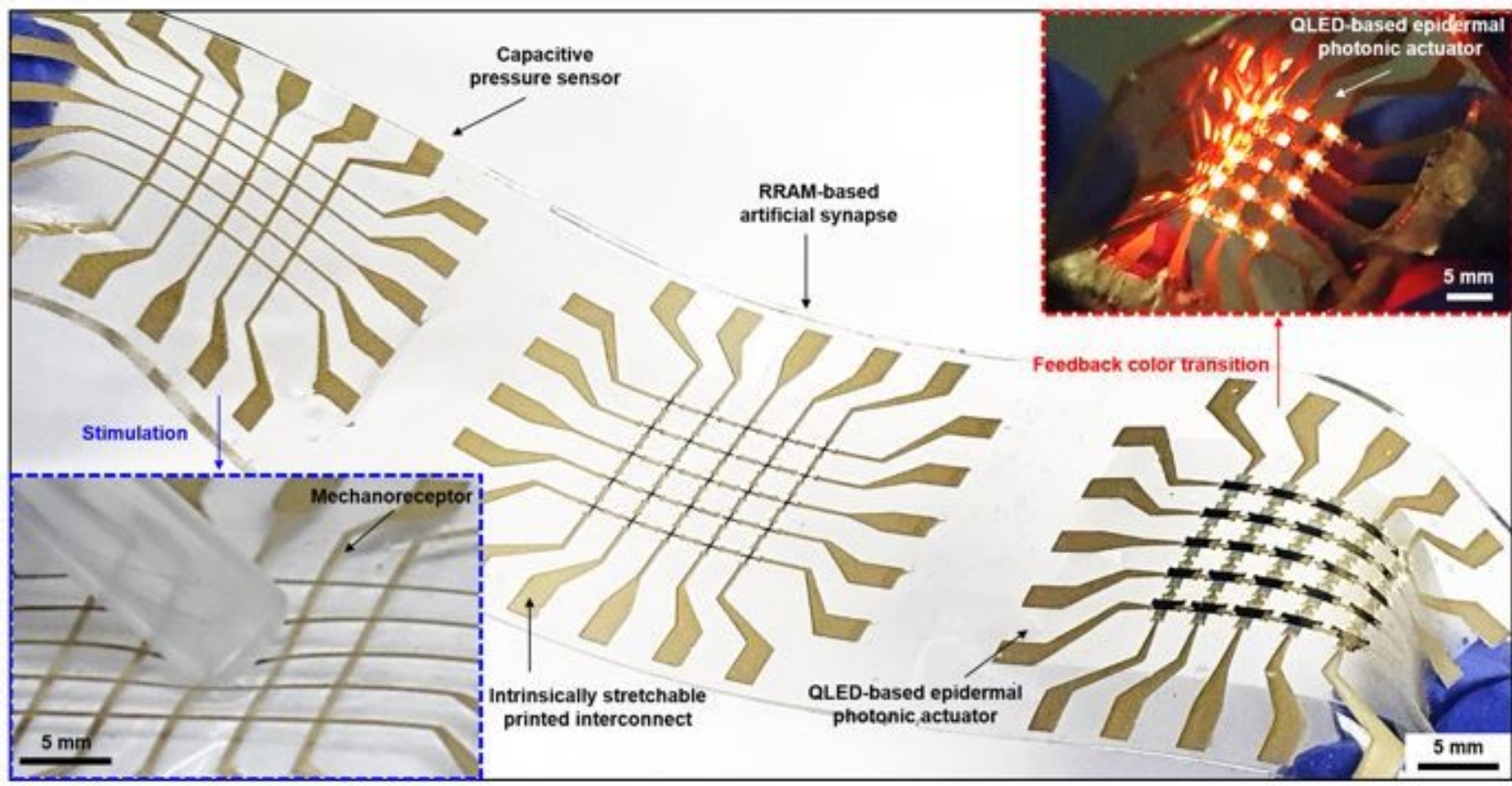

\section{Figure 1}

Golden tortoise beetle-inspired sensory-neuromorphic system. a, Biological recognition system of a golden tortoise beetle stimulated by external pressure. The application of pressure to the golden tortoise beetle changes the receptor potential. The receptor potential induces the synaptic action potential, contributing to information processing in the brain. As a result, the beetle changes the epidermis color for feedback actuation to the external environment. b, Schematic of the bioinspired sensory-neuromorphic system and the corresponding operation process. The system consists of a capacitive pressure sensor (mechanoreceptor), a RRAM (artificial synapse), and a QLED (epidermal photonic actuator). Similar with 
the biological system, the capacitive sensor detects the applied pressure and transmits the signal to the RRAM to change the conductance state by LTP/LTD used for the training/inferencing of the artificial neural network. c, Photograph of the stretchable neuromorphic sensory system. Left inset: Application of stimulation to the mechanoreceptor. Right inset: Feedback color transition of the QLED based on the results of training/inferencing.
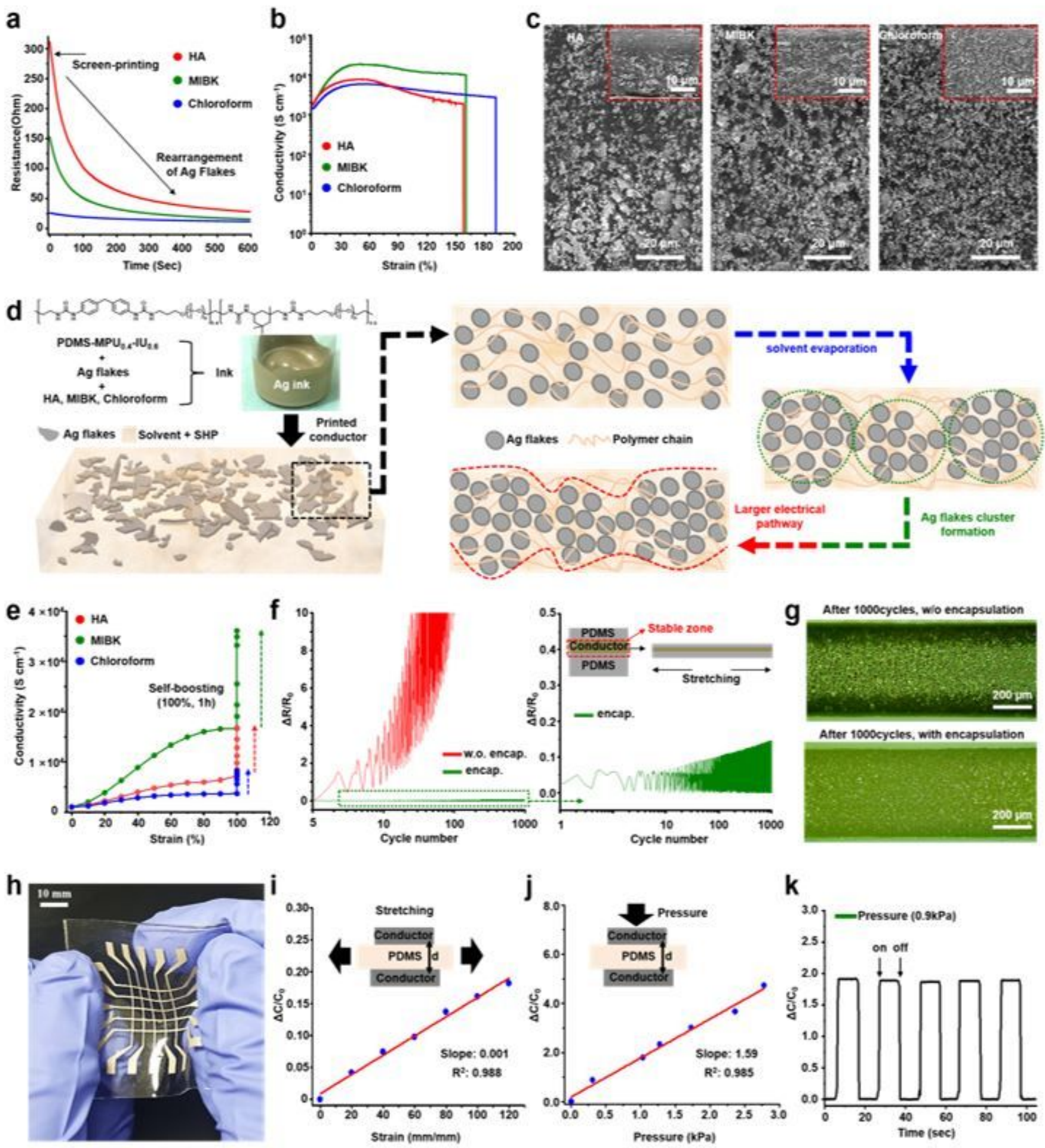

Figure 2 
Characterization of sinter-free printed conductor used for artificial mechanoreceptor fabrication. a, Resistance-time curve of the sinter-free printed conductors formulated using different solvents. $b$, Conductivity-strain graph showing that the conductor formulated with MIBK had the highest conductivity (maximum $\sim 18550 \mathrm{~S} / \mathrm{cm}$ ), while the conductor formulated with chloroform had the highest stretchability (maximum $\sim 191 \%$ ). c, FE-SEM images of printed conductors formulated with (left) HA, (middle) MIBK, and (right) chloroform. A region of extensive aggregation was observed in the HA-based conductor, while homogeneous dispersion was observed for the chloroform-based conductor. In the MIBK-based conductor, Ag flakes were optimally aggregated to afford the highest-conduction path in the polymer matrix. d, Schematic images showing composition of sinter-free printed conductor and process of $\mathrm{Ag}$ flake aggregation in the polymer matrix upon solvent evaporation. After screen-printing of the homogeneous conductive ink (black line), solvent evaporation (blue line) induces the aggregation of $\mathrm{Ag}$ flakes (green line) to form a higher-conductance path in the matrix (red line). e, Conductivity-strain characteristics of each printed conductor (red: HA, green: MIBK, blue: chloroform) showing electrical selfboosting at $100 \%$ strain, with the highest conductivity equaling $36,000 \mathrm{~S} / \mathrm{cm}$. f, Effects of encapsulation on conductor resistance in cyclic test. g, Optical microscopy images showing (top) the formation of cracks on the ink surface and (bottom) the absence of these cracks in the encapsulated conductor. $h$, Photograph of the $5 \times 5$ capacitance sensor array fabricated using the MIBK ink. Relative capacitancestrain plots of the capacitive sensor deformed by i, stretching and j, pressure application. $k$, Cycling stability of the capacitance sensor.
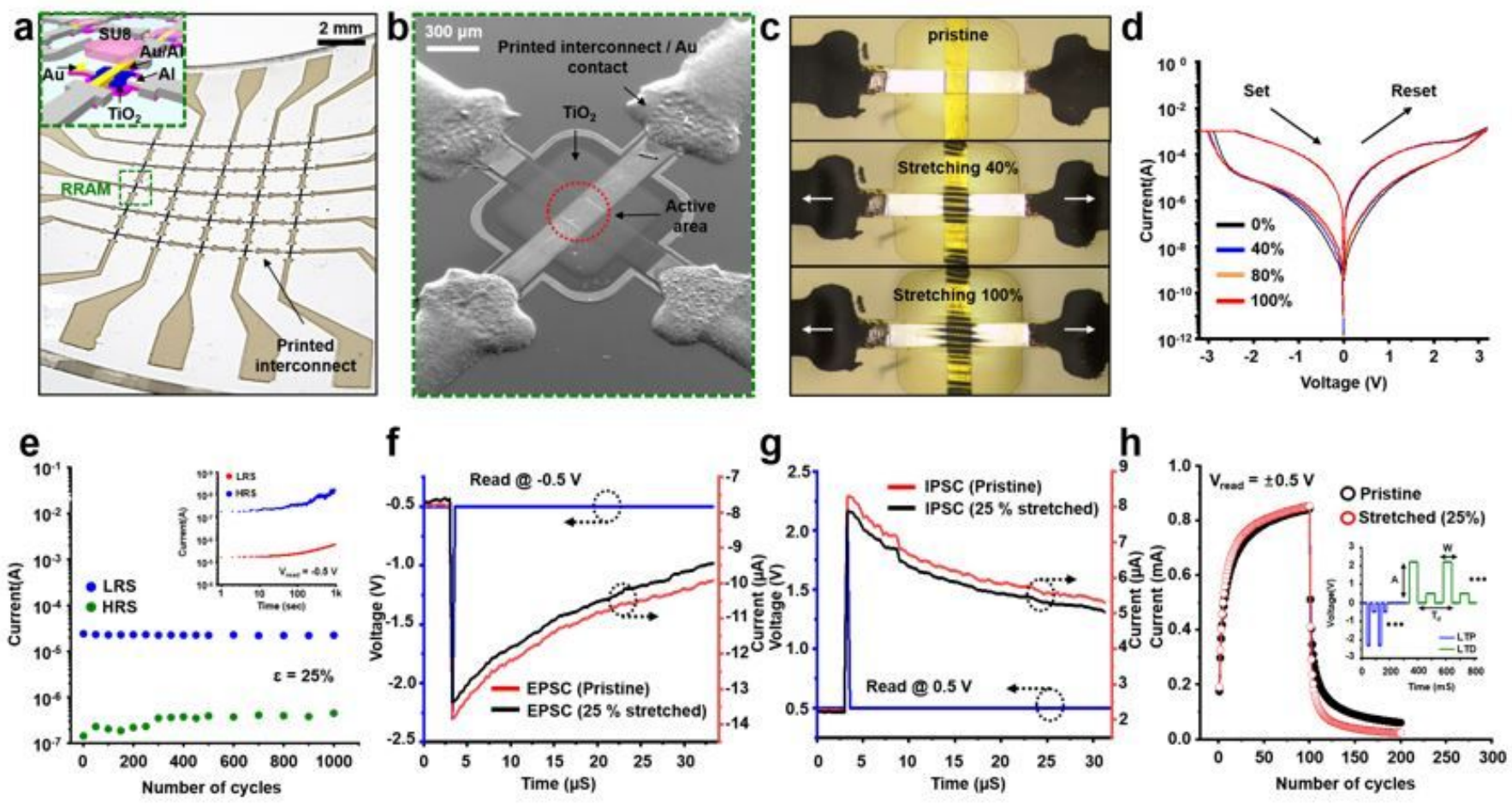

\section{Figure 3}


Characterization of the artificial synapse fabricated using an intrinsically stretchable RRAM. a, Photograph of the intrinsically stretchable RRAM. A device with an Al/TiO2/Al layered structure was fabricated on a PI island and encapsulated by an epoxy polymer (SU-8). b, SEM image of the RRAM island interconnected with a sinter-free printable conductor. The contact Au pad was extended from the bottom Al electrode for low contact resistance. c, Optical microscopy images of the stretched memory ( $100 \%)$ on PDMS. d, I-V characteristics of the stretched memory at different strains (40-100\%). e, Current variations between the low-resistance state (LRS, blue dots) and the high-resistance state (HRS, green dots) at a read voltage of $0.5 \mathrm{~V}$ during 1,000 stretching cycles. $\mathrm{f}, \mathrm{g}, \mathrm{EPSC} / \mathrm{IPSC}$ of our TiO2-based artificial synapse. h, LTP and LTD curves of the artificial synapse in pristine (black circles) and stretched $(\sim 25 \%)$ states (red circles) recorded upon the application of input pulses with an amplitude of $-2.3 \mathrm{~V}$ in LTP and $2.2 \mathrm{~V}$ in LTD, respectively.
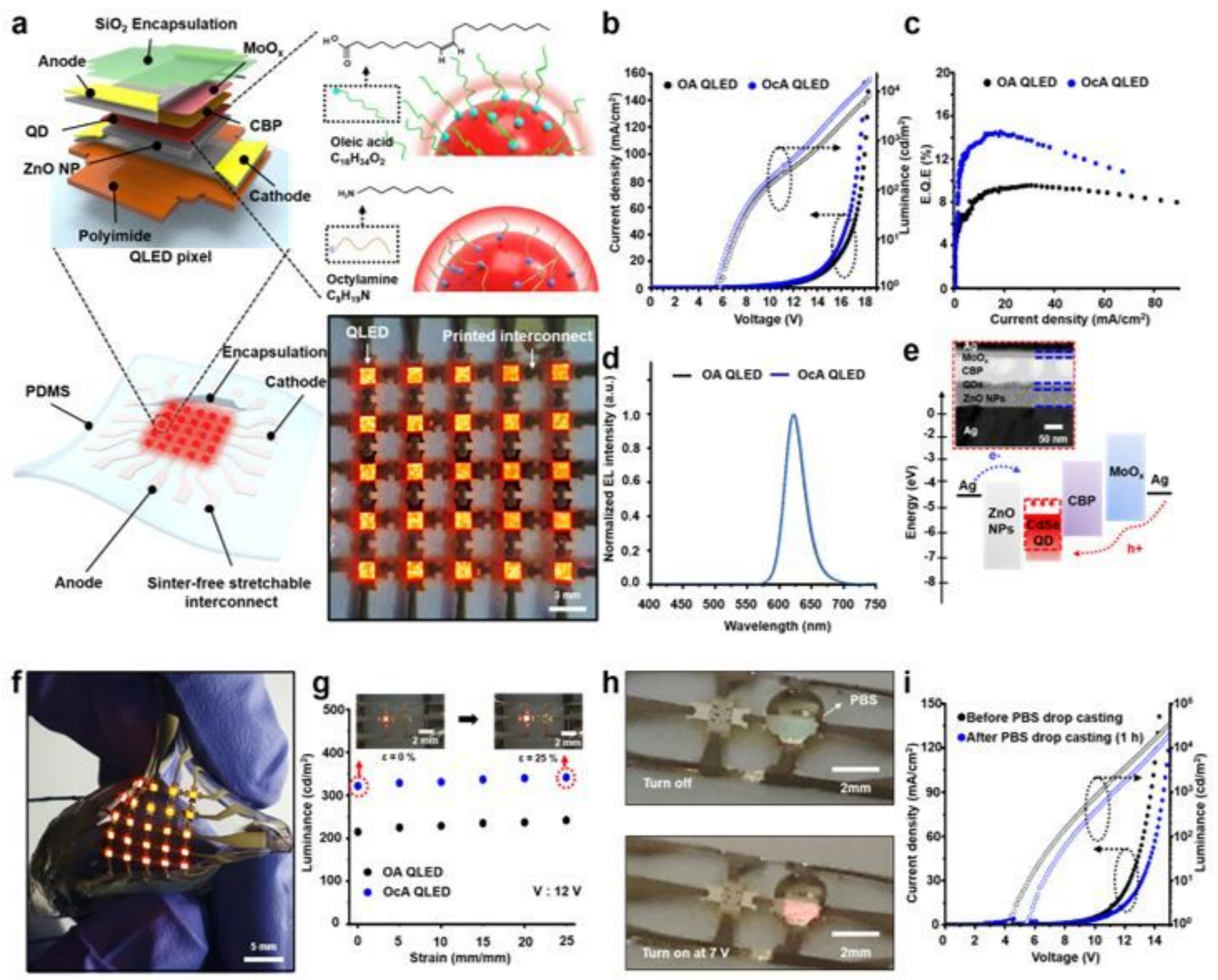

\section{Figure 4}

Characterization of epidermal photonic actuators fabricated using intrinsically stretchable QLEDs. a, Schematic view of epidermal photonic actuators based on intrinsically stretchable QLEDs, showing layer 
information, the magnified QLED pixels from the substrate to the encapsulation layer (top left), a schematic diagram of ligands with OA and OcA (top right), and a photograph of the $5 \times 5$ epidermal photonic actuator on the PDMS substrate (bottom-right inset). b, J-V-L characteristics, c, E.Q.E-current density, and d, normalized EL spectra of a top emissive red CdSe QLED with OA and OcA ligands on a glass substrate. e, Energy diagram of the red CdSe QLED and cross-sectional transmission electron microscope image of the device. $f$, Photograph of the crumpled epidermal photonic actuator. $\mathrm{g}$, Luminance-strain characteristics of single epidermal photonic actuators (operated at $12 \mathrm{~V}$ ) with $\mathrm{OA}$ (black dot) and OcA (blue dot) ligands. Inset shows the photographs of single epidermal photonic actuators at strains of 0 and $25 \%$. h, Optical images of the single epidermal photonic actuator obtained at different applied voltage amplitudes in PBS solution and $\mathrm{i}, \mathrm{J}-\mathrm{V}-\mathrm{L}$ characteristics of the photonic actuator before/after PBS solution drop-casting.
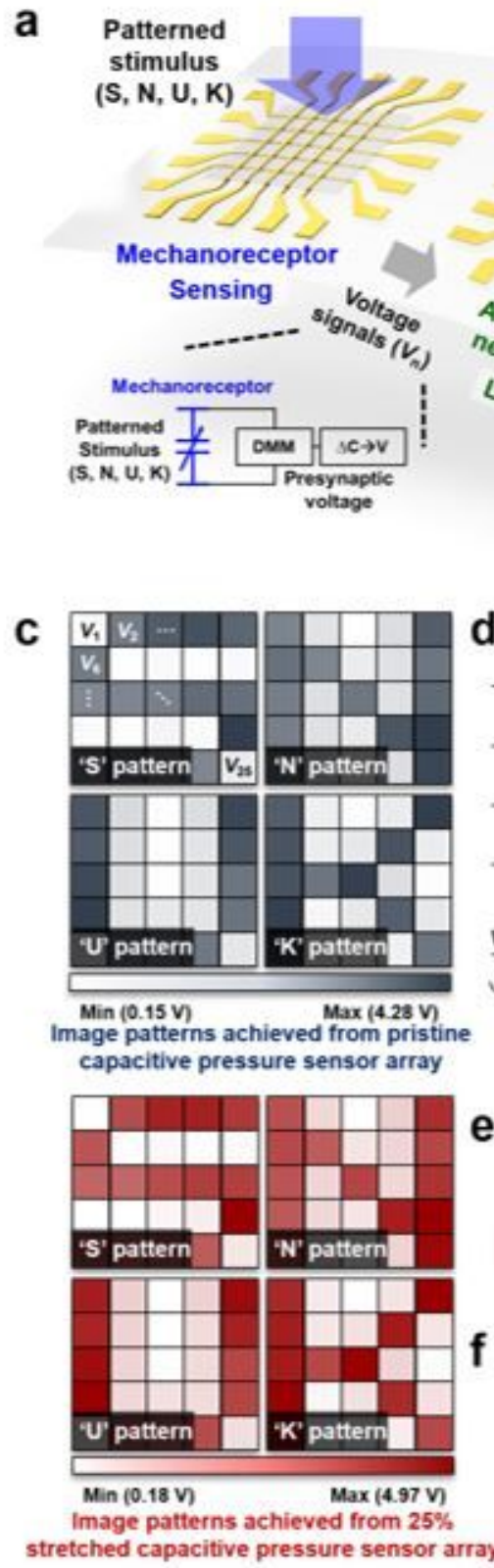
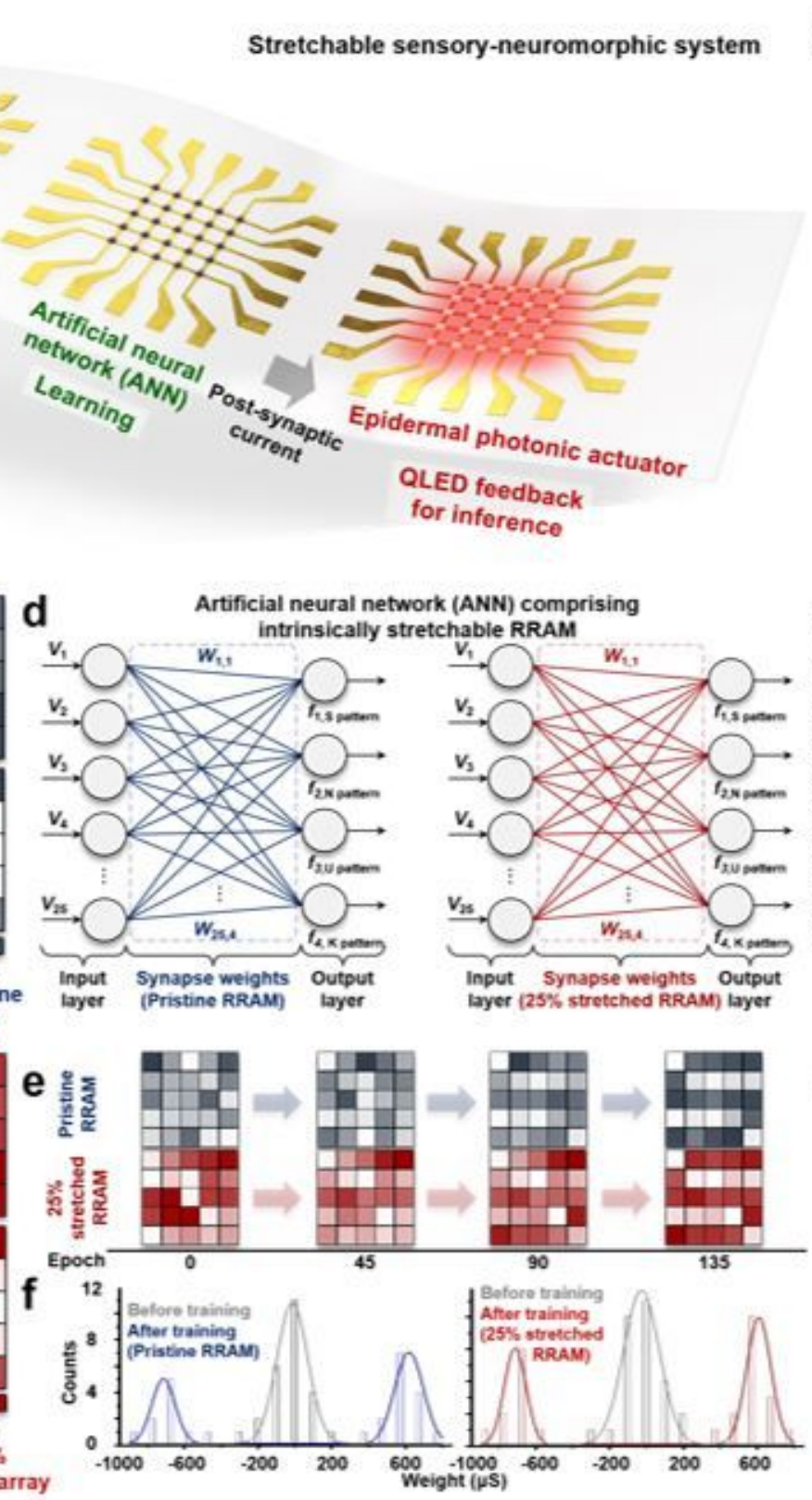
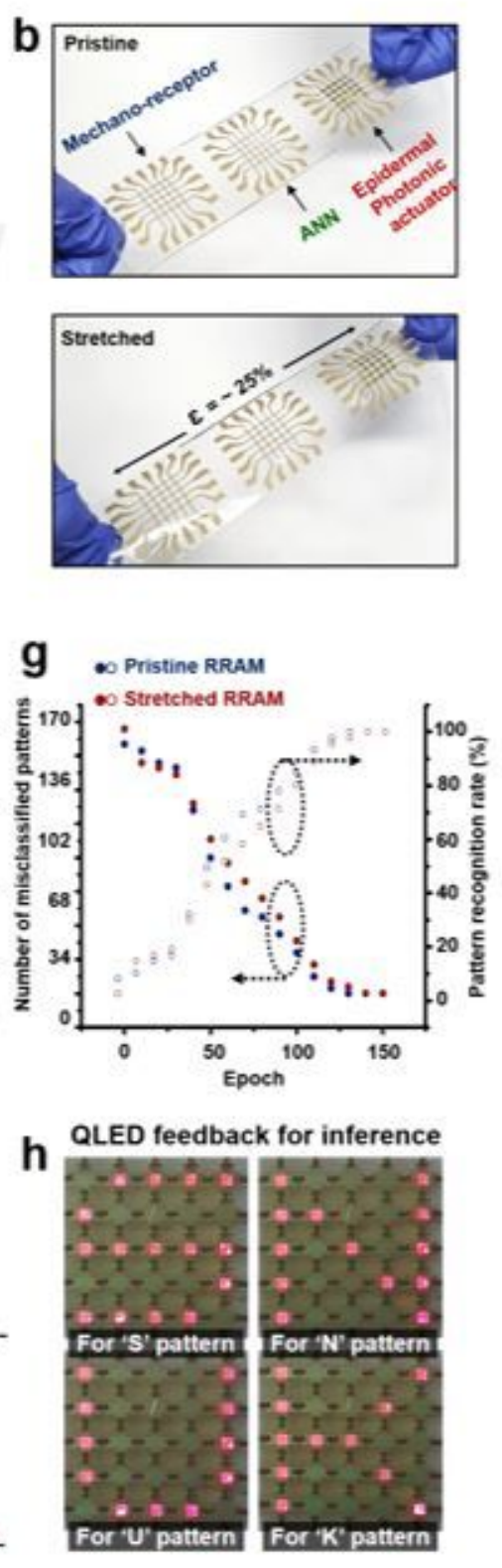
Demonstration of the G-SSNS. a, Schematic of the sensory-neuromorphic system device comprising a mechanoreceptor (for sensing the patterned stimuli and converting capacitance values to voltage signals), an artificial synapse (for training/inferencing from the transmitted voltage signals), and an epidermal photonic actuator (for visualizing the trained/inferenced information). b, Photographs of the GSSNS in pristine (top) and $25 \%$-stretched (bottom) states. c, Image patterns (" $\mathrm{S}$," "N," "U," and "K") provided by the pristine (top) and $25 \%$-stretched (bottom) mechanoreceptors. $d$, Training/inferencing of the neural network in pristine (left) and $25 \%$-stretched (right) states. Each neural network consisted of 25 input neurons corresponding to each pixel, 4 output neurons corresponding to each pattern, and $25 \times 4$ synapses connected to the neurons. e, $\mathrm{f}$, Weight mapping and histogram of synaptic weights connected to $\mathrm{f} 1, \mathrm{~S}$ pattern with respect to the training epoch. $\mathrm{g}$, Calculation of the pattern recognition efficiency and the number of misclassified patterns for every 10 epochs. A $100 \%$ pattern recognition efficiency and no misclassified patterns were obtained after 140 epochs in both pristine and $25 \%$-stretched states. $h$, Photographs of the epidermal photonic actuator array visualizing the trained/inferenced patterns of " $S$ " (top left), “N” (top right), "U” (bottom left), and “K” (bottom right).

\section{Supplementary Files}

This is a list of supplementary files associated with this preprint. Click to download.

- SupplementaryMovie1.mp4

- SupplementaryInformation.docx

- SupplementaryMovie2.mp4

- SupplementaryMovie3.mp4

- SupplementaryMovie4.mp4 